\title{
In celebration of methods
}

As evidenced by the cake adorning the cover, Nature Methods is five years old. To celebrate this anniversary, we look at methodological development and its role in scientific inquiry.

Five years ago on September 29, 2004, the first issue of Nature Methods — the first Nature-branded journal devoted solely to methodological development—went live on nature.com, and 60,000 copies of the journal were winging their way to readers. In celebration, this anniversary issue includes a collection of commentaries from historians and scientists discussing the impact and progress of methodologies in the life sciences.

With Nature Methods we wanted to provide a highprofile publication destination for methods developers and technologists, and an accessible source of information for biologists on important new laboratory methods in the life sciences. This was our small, humble attempt to help redress a historical bias in the publishing field against methods development.

"Too often, discussions of technique and method in science have indeed been seen as signs of a limited imagination-empirical work associated with tinkering, rather than conceptual work associated with theory and discovery," write science historians Angela Creager and Hannah Landecker in a Historical Commentary on page 701. They go on to illustrate-using the examples of tissue culture and radioisotopes - the fallacy of such attitudes and conclude that the distinction between science and technology is artificial. In fact, technical development plays a central role in shaping the progress of modern biology.

The impact of technology on biological research is aptly illustrated by the developments currently underway in sequencing technology. In a Commentary on page 711, Piero Carninci describes how the recent development of RNA sequencing (RNA-seq) using next-generation sequencing technologies is impacting RNA-based research and posits that this may be leading us out of the 'dark ages' of the transcriptome.

Although advances in sequencing technology are bringing enlightenment in regards to genome structure and gene expression, determining how changes in gene expression give rise to phenotypes, whether in single cells or in whole organisms, also requires other technologies. On page 721, Sean Collins, Jonathan Weissman and Nevan Krogan note how advances in various aspects of genetic screening are allowing researchers to characterize the genome at a functional level.

New technologies have the power to drive biological advances, but owing to their novelty and complexity they also have the potential to generate inaccurate data when used improperly. This has been true for a number of modern technologies such as microarrays and mass spectrometry. On page 717, mass spectrometry-based proteomics pioneer Matthias Mann discusses what is needed to realize the enormous potential of this powerful technique for biological understanding through direct assays of proteins. Although technological advances are improving performance, and will continue to do so, there is also a great need for rigorous analysis of the performance itself. These analyses may need to span everything from sample handing and preparation to instrument and algorithm performance, and even data analysis procedures.

The impact of technological and methodological development on the ability of researchers to understand biological function seems indisputable. From the awarding of the Nobel Prize in Medicine to John Enders, Thomas Weller and Frederick Robbins in 1953 for growing poliovirus in tissue culture to last year's prize in chemistry to Osamu Shimomura, Martin Chalfie and Roger Tsien for the discovery and development of GFP, new methodologies and tools have shaped scientific progress.

As Hannah Landecker argues in a Historical Commentary on page 707, techniques development could also be a driving force in what appears to be a cyclic push-pull between static and dynamic or quantitative and qualitative methods of investigation. Indeed, the ascendency of live-cell imaging - driven by the development of intracellular calcium dyes and fluorescent proteins (see Commentary by Michael Davidson and Robert Campbell on page 713) - has spurred technical innovation in microscopy and a recent shift from static quantitative methods to more qualitative dynamic methods.

But also apparent in biological research at present are efforts to provide quantitative frameworks for more exacting observations that could form the basis of biological theories and laws. This is epitomized by the rise of systems biology and automated imaging, both of which strive to extract quantitative data from methodologies that have been historically intractable to such analyses.

The dizzying pace of change in investigative methods available to biological laboratories creates challenges for researchers trying to determine the most suitable techniques for their area of inquiry. We hope that Nature Methods has been helpful in guiding these choices by providing a desirable place to publish and read about the latest and greatest methods and tools. We will strive to grow and evolve along with the methods we highlight so as to better serve our authors and readers for another exciting five years and beyond. 